\title{
On Conducting Tests in Software Engineering Courses during the COVID-19 Pandemic
}

\author{
Pankaj Kamthan \\ Computer Science and Software Engineering \\ Concordia University \\ Montreal, Canada \\ pankaj.kamthan@concordia.ca
}

\begin{abstract}
The circumstances presented by the COVID-19 pandemic have had a severely debilitating impact on education, in general, and software engineering education, in particular. This paper describes the author's experience over three semesters in conducting oral and written tests in six software engineering-related courses with around 500 students overall. The technical as well as non-technical challenges encountered are discussed, and educational lessons based on the reactions and responses of students are given. The proposed concepts, decisions, and processes herein are effectively generalizable and potentially applicable to other types of courses and to (not necessarily pandemic-related) online teaching-related situations in general.
\end{abstract}

Keywords-collusion and plagiarism; conceptual model; distance education; e-learning; formative and summative assessment; online teaching and learning

\section{INTRODUCTION}

The COVID-19 pandemic was an unprecedented, disruptive event of 2020 that continues to have severe ramifications on a global scale in 2021. To prevent infections, to mitigate and control the rate of transmission, and to minimize hospitalizations, the governments everywhere have been compelled to declare community lockdowns, and to closing or restricting access to all those sectors of society that necessitate a large number of people congregating in closed spaces for a prolonged period of time, including educational institutions [1]. In order to continue to remain operational, the educational institutions have inexorably resorted to a suitable combination of e-learning, distance education, and online teaching and learning [2]. This paper reports the experience at a University of conducting tests in six courses over three semesters, Spring 2020, Summer 2020, and Fall 2020, in such an environment. These courses are part of an undergraduate program (namely, Introduction to Software Engineering and Object-Oriented Methods with UML), and a graduate program (namely, Advanced Software Architectures, Software Comprehension and Maintenance, Software Engineering Development Processes, and Software Measurement), topics of which are aligned with a taxonomy of software engineering education [3].

The rest of the paper is organized as follows. In Section II, relevant background is provided and related work is discussed. The specifics of the tests, along with associated challenges and lessons learned are presented in Section III. In Section IV, directions for future research are outlined briefly. Finally, in Section $\mathrm{V}$, concluding remarks are given.

\section{BACKGROUND AND RELATED WORK}

The COVID-19 pandemic has obligated teachers from around the world, at every level, in all disciplines, to improvise and innovate in order to continue to provide an acceptable level of education. The notions of e-learning, distance education, and online teaching and learning have existed for quite some time. Indeed, they have been practiced to varying degrees over the years, especially since the inception of the Web, and their advantages and disadvantages are known $[4,5]$. However, they have garnered special attention, assumed renewed significance, and seen a broad adoption during the COVID-19 pandemic.

The sudden transition from offline to online teaching has led to, somewhat expectedly, mixed results for administrators, teachers, and students $[6,7,8,9]$. In [10], the experience, both before and after the declaration of COVID-19 as a pandemic, of conducting tests using MyElearning in a digital electronics course is described. In [11], the experience of conducting a test comprising multiple choice questions and a case study-based question using Google Forms, Google Drive, and WhatsApp in a multimedia systems course has been given. In [12], issues related to conducting unproctored online tests using a question bank and Examplify in a general chemistry course are explored. There have been relatively few detailed reports specific to test procedures, processes, and practices during the COVID-19 pandemic, and that, in part, forms the motivation for this paper.

\section{THE TESTS}

The courses in question had a number of components of assessment, including tests. The tests were conducted in alignment with the principles of online teaching [13].

\section{A. Students}

In some cases, the students were geographically distributed across different cities within the same country (Canada) and, in other cases, across different cities in different countries (Canada, China, France, India, and Morocco). In order to prepare the students for oral and written tests, they were (1) told that the standard of questions and problems would be same as in an offline teaching environment, and (2) given sample questions/answers and problems/solutions, as the case may be, well in advance so that they could practice and familiarize themselves with the unique style of the upcoming tests. The students were either accustomed to the technologies and tools used for the tests, or learned them readily on their own.

DOI reference number: 10.18293/SEKE2021-025 


\section{B. Technology and Tool Environment}

The technologies and tools for the tests were provided by the University, and were deemed suitable for online tests [14]. The syllabus, schedule, and other pertinent details of the tests were announced on Moodle, a course management system. The tests were conducted in real-time using Zoom, a cloud-based video communications software-as-a-service (SaaS). The students submitted their written tests on a special-purpose electronic assignment submission system (EAS) supported by the University. The access of each of these systems required a username-password-based authentication. The students were given the alternative to submit their tests via e-mailing the teacher in the event that a submission on the EAS was not possible for technical reasons (say, if the system was unavailable because it was overloaded or was non-operational).

\section{Oral Tests}

The COVID-19-related regulations of the University were such that they allowed the students to attend the lectures without using any audio or video on their side. (The students were given the right to turn their camera and/or microphone off during the lectures, and many did exercise that right.) The purpose of the oral test was, therefore, not only about an assessment of their verbal aptitude or ability to answer technical questions in a relatively short period of time, but also to improve personal rapport between the teacher and the students, by being able to see them, speak to them, and hear them 'in person', in real-time.

1) Description of the Prerequisites, Regulations, and the Process of Oral Tests

There was one oral test per course. The duration of the test, depending on the class size, lasted between 2 hours to 8 hours. A pool of questions was prepared in advance of the test. To reduce repetition and to decrease the possibility of students who were tested earlier to help those who were tested later, the number of questions exceeded the class size. The questions were either open or closed, but mostly closed for which answers were also prepared in advance of the test. The teaching assistants were consulted to ensure that the questions were within scope of the syllabus and answerable within the time allowed. The examiners during the test included the teacher and all the teaching assistants for that course. Each student was given a specific time slot of 10 -minute duration. The students were prohibited from entering a time slot not allotted to them, as that would distract the students who were scheduled and expected to be present during that slot. There was a small penalty if they did, and some in fact did. (This would be overcome in due course by the use of Zoom's 'Waiting Room' feature.) In cases where the class size was rather large, which would make the overall duration of the oral test prohibitively long, multiple students were given the same time slot.

The process for the oral test was similar to an interview process, and is shown in Fig. 1 as a UML Activity Diagram with swimlanes representing the actors involved. Each student was asked 3 to 4 questions. The students in the same time slot were usually asked different questions, unless one of the students could not answer a question, at all or properly, in which case the same question was posed to another student in the same time slot. The examiners rotated in asking questions. The initiator of a question (namely, the teacher or one of the teaching assistants) was pseudo-random (that is, not predetermined). The students were not allowed to revisit a question that they could not answer earlier. This was done to prevent students seeking external help, such as asking someone or checking the Web for a possible answer. The session was recorded in case a student may wish to revisit answers that the student provided, and some students did. The marks were determined and recorded in real-time by the examiners.

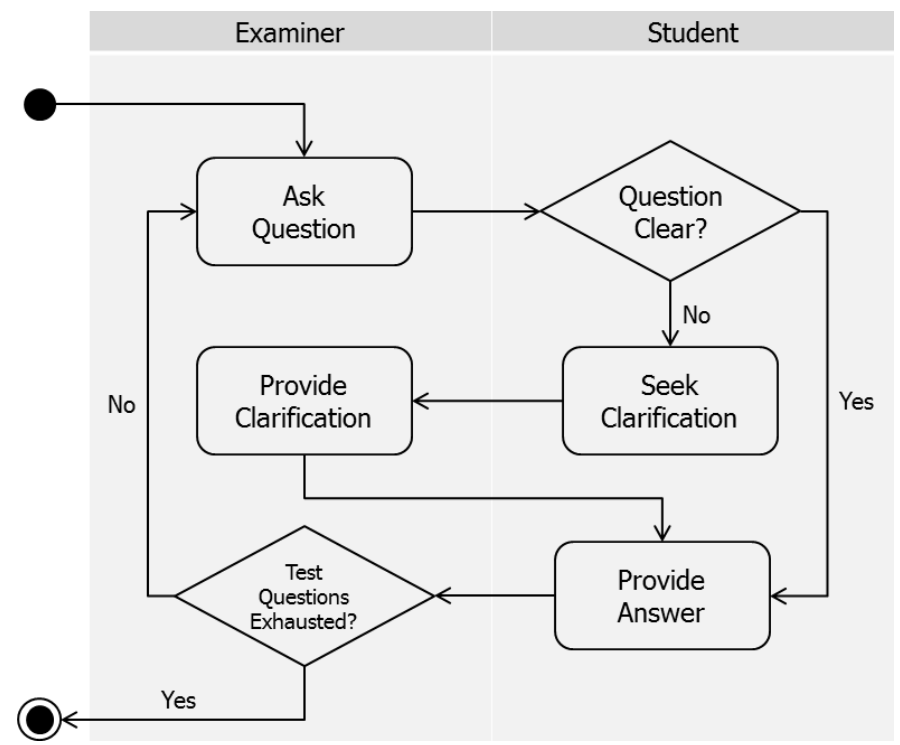

Figure 1. A UML Activity diagram for the oral test process.

\section{2) Challenges and Lessons Learned from Oral Tests}

- Scope of Questions. The questions did not include any that would require the need for calculating, diagramming, or programming. In case of a question with an open answer, some students attempted to give a rather lengthy, albeit correct, answer. For consideration of time, such students had to be interrupted and were told to stop speaking to allow sufficient time for other questions (and/or for other students to have their turn to be asked).

- Understanding Questions. In some cases, the students had difficulty listening to a question, perhaps due to technical (audio) problems or due to the way it was read and spoken by an examiner. In such cases, the question was repeated, verbatim or with a slight variation.

- Quality of Responses. The quality of responses varied considerably among the students. There were primarily three types of problems: (1) incorrect or partially incorrect answer, (2) answer not matching the question, and (3) over-answering (correct answer that was annotated with extraneous statements that were not always correct). It is unclear whether those students whose verbal responses was deemed problematic would have said or done anything different if they had more time, if were allowed to express their answers in writing, or if they were in an offline teaching environment.

- Student Anxiety. There were some students who had never been in an interview or given an oral test previously, 
and were therefore noticeably, as their facial expression and/or body language implied, unusually anxious.

- Duration of Test. The duration of the test was not an issue for the students, but was somewhat exhausting for the examiners in those cases where it exceeded 4 hours.

- Collusion and Plagiarism. In spite of the steps taken to prevent collusion or plagiarism, it did appear from the nature and/or the promptness of the answers to non-trivial questions that some students either attempted to collude/plagiarize, or may even have colluded/plagiarized successfully. (For example, in a few cases, the students appear to look elsewhere for an unusually long period of time as if they were staring at another person or at a different screen.) However, in absence of concrete evidence, no cases were pursued. In case of a suspicion, the responses given by the students to the original question were dismissed, and they were asked an 'extra question' after being reminded to look directly at the camera at all times while answering.

\section{Written Tests}

1) Description of the Prerequisites, Regulations, and the Process of Written Tests

There were two written tests per course. The tests assumed that the students had access to (at least) the lecture notes, a calculator, a dictionary, Zoom, and the ability to upload documents to the EAS. The students were provided with a Microsoft Word or LATEX template, depending on the course, and were expected to give a solution to each problem in the template provided. The students were told that the problems would be based strictly on the syllabus specified, and, to discourage the use of sources that were not permitted on the test, the solutions that included claims based on querying a public search engine would not get credit. To encourage the students to make proper use of the time allowed, they were also told to place attention on quality, not quantity, in writing, and that, in general, they should avoid overwriting. The students were also told that for each problem, certain space and certain time were allocated, and that they should manage both space and time. The students were told that, in general, they were not to communicate, including seek clarification on a problem, as the ability to read and understand the problems given was part of the test. However, in case of an emergency, they could communicate privately with the teacher either by e-mail or by using the chat feature of Zoom. Finally, the students were told that it would be in their interest to have their technical environment ready in advance of the test. This included Zoom running, Microsoft Word template open, and the EAS URL in the Web user agent entered before the test commenced.

The process for the written test is shown in Fig. 2 and Fig. 3 as a collection of UML Activity Diagrams from the perspectives of the examiner and the student, respectively. In Fig. 2, $\mathrm{T}$ is the time allocated for a problem on a particular page/screen. The URL for a rendezvous on Zoom was announced privately (rather than publicly, for security reasons) on Moodle. The tests had 20 to 24 problems each, depending on the course. In each test, every problem was allocated a certain amount of time, which ranged from 2 to 6 minutes. The tests proceeded as follows. At the start of a test, a screen rendering a PDF document was shown with a problem statement and the duration for that problem. There was one problem per page (and, therefore, per screen). For each problem, the students were given a 1-minute verbal reminder before the duration of that problem lapsed. Upon the lapse of duration for a given problem, the screen was shifted to the next problem, in a sequential manner. There was no backtracking, meaning it was not possible for the students to go back to any of the problems shown previously. At the end of the test, the students were to upload their tests on the EAS, and everyone did. There was per-minute penalty for a late submission, regardless of the reason, and some students were penalized.

The tests were not proctored, despite the possibility. The students were not required to have their camera on, and none of them did. To avoid disturbing the others taking the test, the students were also told not to use their microphone under any circumstance, and, again, none of them did.

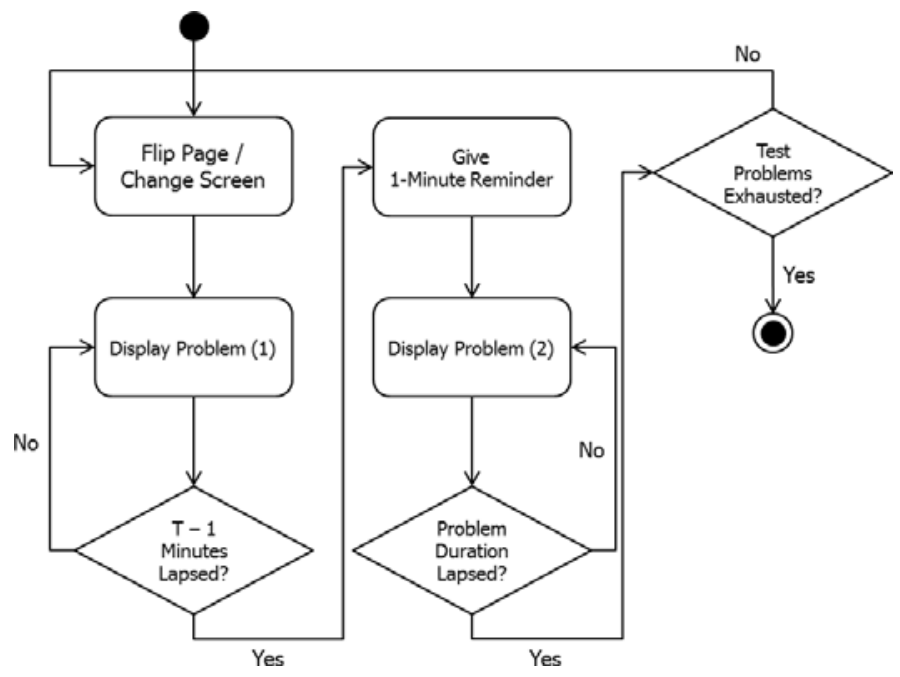

Figure 2. A UML Activity diagram for the written test process from an examiner's perspective.

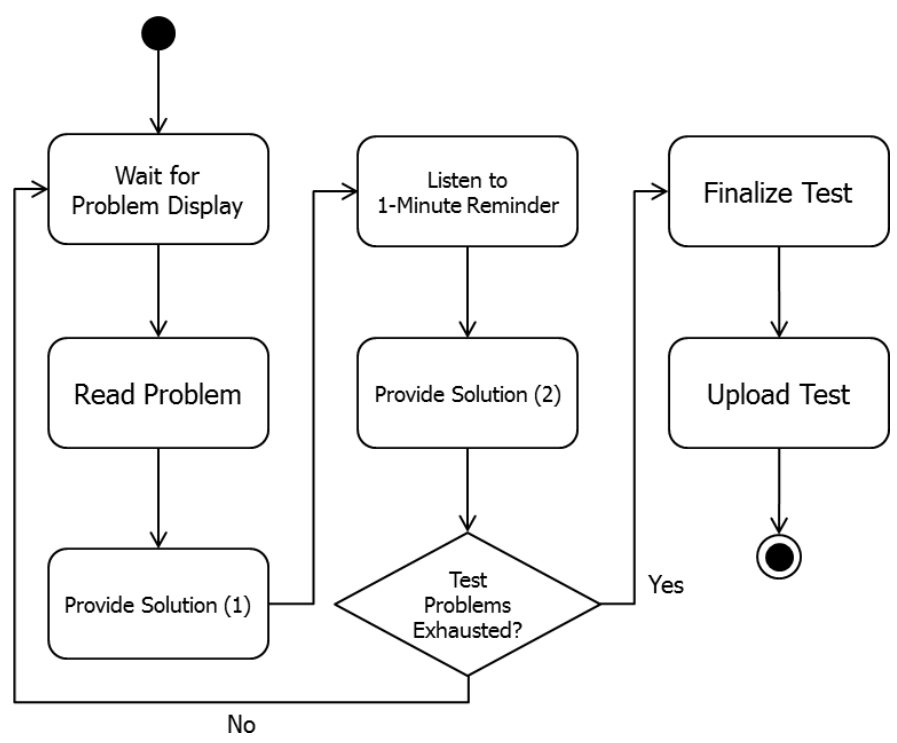

Figure 3. A UML Activity diagram for the written test process from a student's perspective. 
2) Challenges and Lessons Learned from Written Tests

- Problems Requiring Mathematical Notation. The problems required solutions with only simple and short mathematical expressions, if at all, as typesetting mathematics can be tedious and rather time consuming.

- Quality of Writing. The quality of writing varied considerably among the students. It is unclear whether those students whose writing was deemed problematic would have done anything different if they had more time or if they were in an offline teaching environment.

- Quality of Diagramming. The students were informed in advance that they may need to draw simple UML Class Diagrams, UML Component Diagrams, and/or UML Use Case Diagrams. The syntactic, semantic, and/or pragmatic quality of the diagrams varied, primarily because of the time allocated to problems that required diagramming was, as self-reported by the students, deemed insufficient.

- Weak Internet Connection. There were a few students who were on a Wi-Fi connection that, as self-reported by those students, turned out to be not reliable enough for the tests. These students had to repeatedly reconnect to Zoom.

- Students with Disabilities. The University regulations require that the students who are registered with the Access Centre for Students with Disabilities (ACSD) be given special accommodation during the tests. In particular, the students registered with the ACSD are, in some cases, given extra time for the tests, where the exact extra time is on a per case basis, depending on the student. In some of the courses, there were students registered with the ACSD, and, for such students, the test process was repeated with a longer than usual duration.

- Collusion and Plagiarism. In spite of the steps taken to prevent collusion and plagiarism, there were a few cases where, as the evidence suggested clearly, some students got together in small groups and copied from each other. These cases were duly identified, pursued, and reported.

\section{E. Discussion}

The students were solicited to provide feedback on their experiences, with the tests and otherwise, in the courses. There were no objections from the students regarding the fairness of any of the tests. The nature of the types of problems or the differences in time zones did not seem to have any notable impact on the performances of the students in any of the tests.

\section{DiRECTIONS FOR FUtURE RESEARCH}

There are several established ways of, as well as technical environments for, conducting an online test $[4,14]$. It could be useful to analyze and compare the degree of comfortableness, individual as well as communal behavior, performance, and the extent of learning of students in written tests as described in this paper with the same or similar group of students (1) in an offline teaching environment, (2) using a different style of question/answer or problem/solution methodology, and/or (3) using a different set of technologies and tools, and is therefore of research interest.

\section{CONCLUSION}

The obstacles presented by the COVID-19 pandemic to (software engineering) education go beyond that of those typical of 'conventional' distance education or online teaching and learning. However, this paper has shown that using only basic technologies and tools, it is possible to conduct verbal and written tests, with acceptable outcomes, albeit with associated challenges, some of which could, hopefully, be overcome in due course with better planning and preparation.

\section{ACKNOWLEDGMENT}

This paper is dedicated to the indelible memories of Terrill Fancott and Peter Grogono, who passed away in 2020 and 2021, respectively. They inspired and guided the author over the years in many matters related to software engineering education. The author is grateful to CUPFA for a Professional Development Grant and to his teaching assistants for useful discussions.

\section{REFERENCES}

[1] E. M. Onyema, N. C. Eucheria, F. A. Obafemi, S. Sen, F. G. Atonye, A. Sharma, and A. O. Alsayed, Impact of Coronavirus Pandemic on Education. Journal of Education and Practice, 11(13): 2020, 108-121.

[2] J. L. Moore, C. Dickson-Deane, and K. Galyen, E-Learning, Online Learning, and Distance Learning Environments: Are They the Same? Internet and Higher Education, 14: 2011, 129-135.

[3] S. Pizard and D. Vallespir, Developing a Taxonomy for Software Engineering Education through an Empirical Approach. CLEI Electronic Journal, 23(2): 2020, 1-23.

[4] A. W. Bates, Teaching in a Digital Age: Guidelines for Designing Teaching and Learning, Second Edition. Tony Bates Associates, 2019.

[5] A. Alenezi, The Role of e-Learning Materials in Enhancing Teaching and Learning Behaviors. International Journal of Information and Education Technology, 10(1): 2020, 48-56.

[6] M. H. Rajab, A. M. Gazal, and K. Alkattan, Challenges to Online Medical Education during the COVID-19 Pandemic. Cureus, 12(7): 2020, 1-11.

[7] H. K. Al-Mohair and S. Alwahaishi, Study on Students' Experiences about Online Teaching during COVID-19 Outbreak. Technium Social Sciences Journal, 8: 2020, 102-116.

[8] S. Motogna, A. Marcus, and A.-J. Molnar, Adapting to Online Teaching in Software Engineering Courses. The Second ACM SIGSOFT International Workshop on Education through Advanced Software Engineering and Artificial Intelligence (EASEAI 2020), Virtual Event, USA, November 9, 2020.

[9] N. Tuaycharoen, University-Wide Online Learning during COVID-19: From Policy to Practice. International Journal of Interactive Mobile Technologies, 15(2): 2021, 38-54.

[10] D. Idnani, A. Kubadia, Y. Jain, and P. Churi, Experience of Conducting Online Test during COVID-19 Lockdown: A Case Study of NMIMS University. International Journal of Engineering Pedagogy, 11(1): 2021, 49-63.

[11] M. L. George, Effective Teaching and Examination Strategies for Undergraduate Learning during COVID-19 School Restrictions. Journal of Educational Technology Systems, 49(1): 23-48, 2020.

[12] T. M. Clark, C. S. Callam, N. M. Paul, M. W. Stoltzfus, and D. Turner, Testing in the Time of COVID-19: A Sudden Transition to Unproctored Online Exams. Journal of Chemical Education, 97: 2020, 3413-3417.

[13] A. W. Bangert, The Seven Principles of Good Practice: A Framework for Evaluating On-Line Teaching. Internet and Higher Education, 7: 2004, 217-232.

[14] L. Dawley, The Tools for Successful Online Teaching. Idea Group, 2007. 